\title{
As espécies de Gastrotheca Fitzinger na Serra dos Órgãos, Estado do Rio de Janeiro, Brasil (Amphibia: Anura: Amphignathodontidae)
}

\author{
Eugenio Izecksohn \& Sérgio P. de Carvalho-e-Silva
}

Instituto de Biologia, Universidade Federal do Rio de Janeiro. Caixa Postal 68044, 21944-970 Rio de Janeiro, Rio de Janeiro, Brasil. E.mail: eugenio@izecksohn.com.br; sergio@biologia.ufrj.br

\begin{abstract}
The species of Gastrotheca Fitzinger at Organs Mountains, Rio de Janeiro State, Brazil (Amphibia: Anura: Amphignathodontidae). A study of the anurofauna of the Brazilian rainforest in the mountain chain of Serra dos Orgãos (state of Rio de Janeiro) revealed the occurrence of three species of Gastrotheca Fitzinger, 1843: G. albolineata (Lutz \& Lutz, 1939), G. ernestoi Miranda-Ribeiro, 1920 and G. fulvorufa (Andersson, 1911). These taxa are compared with respect to their external and cranial morphology, as well as call structure, and diagnosed as three unique species. The name Gastrotheca microdiscus (Andersson, 1910), which G. fulvorufa and G. ernestoi have been recently considered as synonyms, is not applied to either of the three species from Serra dos Órgãos, given the smaller size of the disks of fingers and toes and by the derm of the head completely involved in a faintly rugose cranial ossification in G. microdiscus, as originally described. Gastrotheca viridis Lutz \& Lutz, 1939 is herein considered a synonym of Gastrotheca ernestoi.

KEY WORDS. Taxonomy; Southeastern Brazil; Gastrotheca fulvorufa; Gastrotheca ernestoi; Gastrotheca albolineata.
\end{abstract}

RESUMO. O estudo dos anfíbios que ocorrem nas florestas da Serra dos Órgãos, no Estado do Rio de Janeiro, sudeste do Brasil, resultou no registro de três espécies de Gastrotheca Fitzinger, 1843: G. albolineata (Lutz \& Lutz, 1939), G. ernestoi Miranda-Ribeiro, 1920 e G. fulvorufa (Andersson, 1911). Essas espécies são comparadas, inclusive com exame dos crânios e da vocalização, e reconhecidas como diferentes. O nome Gastrotheca microdiscus (Andersson, 1909), ao qual G. fulvorufa e G. ernestoi têm sido consideradas sinônimos por alguns autores, é tido aqui como inaplicável para qualquer dessas três espécies devido ao tamanho menor dos discos dos dedos e artelhos e pela derme da cabeça completamente envolvida em uma ossificação craniana debilmente rugosa em G. microdiscus, como consta da descrição original daquela espécie. Gastrotheca viridis Lutz \& Lutz, 1939 é sinonimizada a G. ernestoi.

PALAVRAS-CHAVE. Taxonomia; sudeste do Brasil; Gastrotheca fulvorufa; Gastrotheca ernestoi; Gastrotheca albolineata.

As espécies de Gastrotheca Fitzinger, 1843 são características por suas fêmeas apresentarem uma bolsa dorsal com abertura posterior onde os ovos se incubam, sendo que ao menos 52 espécies ocorrem na América do Sul, Panamá e Costa Rica (Frost 2007). As espécies brasileiras conhecidas devem apresentar desenvolvimento direto a julgar pelo tamanho de seus ovos (BOULENGER 1888, Anderson 1910, Miranda-Ribeiro 1920, 1926, Izecksohn \& CarvaLHO-e-Silva 2001, SaChSSE et al. 1999). Exemplares dessas espécies são relativamente escassos nas coleções dos museus em decorrência de seus hábitos, pois normalmente se mantêm e se reproduzem no alto de árvores, na floresta atlântica, e raramente são encontrados ao alcance dos colecionadores. Em decorrência disto, tais espécies têm sido descritas com base apenas em um ou dois exemplares e ainda são mal conhecidas quanto ao significado da variabilidade de seus caracteres, o que tem acarretado, por um lado o surgimento de sinônimos, e por outro, sinonímias que parecem ser incorretas diante dos caracteres referidos nas descrições originais das espécies. Assim, apesar de terem sido reunidos na literatura seis nomes atribuíveis a espécies brasileiras de Gastrotheca, após Cochran (1955) haver colocado as espécies Nototrema fulvorufa Andersson, 1911 e Hyla parkeriana DeWitte, 1930 na sinonímia de Gastrotheca microdisca [sic] (Andersson, 1909), e Duellman (1984) ter acrescentado à lista de sinônimos daquela espécie Gastrotheca ernestoi Miranda-Ribeiro, 1920 e Gastrotheca viridis Lutz \& Lutz, 1939 apenas duas espécies brasileiras permaneceram consideradas válidas: G. microdiscus e $G$. fissipes (Boulenger, 1888). Uma outra espécie, contudo, descrita originalmente como Hyla albolineata Lutz \& Lutz, 1939, baseada em um indivíduo juvenil obtido em Teresópolis, no Estado do Rio de Janeiro, foi posteriormente transferida para o gênero Gastrotheca por SACHSSE et al. (1999) que obtiveram uma fêmea com a bolsa dorsal contendo ovos, de onde eclodiram 16 rãzinhas. IZECKSOHN \& CARVALHO-E-SILVA (2001) não aceitaram a sinonímia de G. fulvorufa e G. ernestoi com G. microdiscus.

Revista Brasileira de Zoologia 25 (1): 100-110, March, 2008 


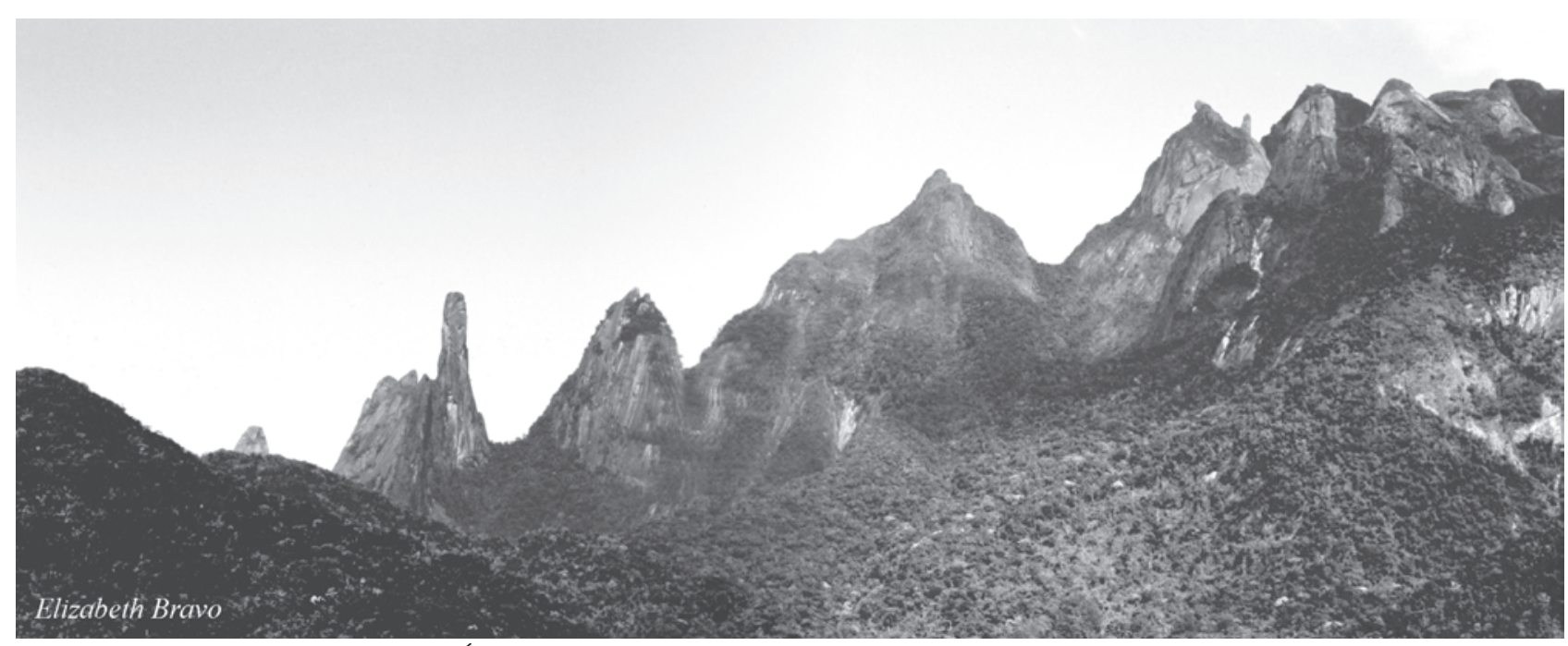

Figura 1. Vista de trecho da Serra dos Órgãos.

A Serra dos Órgãos (senso restrito) (Fig. 1) é um segmento da Serra do Mar, no Estado do Rio de Janeiro, que se limita com a Serra de Macaé ao leste e com a Serra da Estrela ao oeste, se bem que alguns autores considerem, em um sentido mais amplo, os três segmentos reunidos sob o nome Serra dos Órgãos. A região abriga importantes unidades de proteção integral do meio ambiente, podendo ser destacadas, pelas extensões de suas áreas, o Parque Nacional da Serra dos Órgãos, com 10653 ha, e o Parque Estadual dos Três Picos, com 46350 ha. Ao oeste, na vizinha Serra do Tinguá, encontra-se a Reserva Biológica de Tinguá, com extensão de 26136 ha. As encostas cobertas por florestas distribuem-se, nessas montanhas, desde poucas dezenas de metros sobre o nível do mar até altitudes acima de 2000 m. A região foi escolhida como limitação da área de pesquisa para este trabalho, pois além de oferecer facilidades de locomoção, abriga ao menos três distintas espécies de Gastrotheca.

O objetivo deste trabalho é reavaliar sinonímias entre espécies descritas para as regiões Sudeste e Sul do Brasil. O nome G. microdiscus não é aqui aplicado às espécies encontradas na Serra dos Órgãos por razões apresentadas adiante (ver Discussão). Para melhor diferenciar essas espécies, são acrescentados aos caracteres empregados por autores anteriores, outros ainda não utilizados como o aspecto dos frontoparietais e a vocalização.

\section{MATERIAL E MÉTODOS}

O material examinado está depositado nas seguintes coleções: ZUFRJ (Coleção do Laboratório de Anfíbios e Répteis, Instituto de Biologia, Universidade Federal do Rio de Janeiro, Rio de Janeiro), EI (Coleção Eugenio Izecksohn, Instituto de Biologia, Universidade Federal Rural do Rio de Janeiro, Seropédica), RURAL (Coleção do Laboratório de Zoologia do Instituto de Biologia da Universidade Federal Rural do Rio de Janeiro, Seropédica), UNIRIO (Coleção da Universidade Federal do Estado do Rio de
Janeiro), MNRJ (Coleção do Setor de Herpetologia do Museu Nacional, Rio de Janeiro), AL (Coleção Adolpho Lutz, Museu Nacional, Rio de Janeiro) e MZUSP (Coleção de Anfíbios do Museu de Zoologia, Universidade de São Paulo, São Paulo).

As vocalizações foram gravadas em Teresópolis, Estado do Rio de Janeiro, em fitas cassete, com auxílio de um gravador Sony WM-DC6 e um microfone Sennheiser ME-66. As vozes foram digitalizados a 44,1 kHz, 16 bits e analisadas em microcomputador iMac G3 com auxílio do programa Canary (v.1.2.4) ou em microcomputador PC com o programa Raven (v.1.2.1) ambos do Cornell Laboratory of Ornithology (Bioacoustics Research Program). As descrições e análises das vocalizações seguem Weber et al. (2005).

Os crânios foram preparados segundo Izecksonn et al. (2005).

\section{RESULTADOS}

\section{Gastrotheca albolineata (Lutz \& Lutz, 1939)}

Figs 2, 3, 13, 16, 19, 22, 26, 27, 32

Hyla albolineata Lutz \& Lutz, 1939: 69. Localidade-tipo: Teresópolis, Estado do Rio de Janeiro, Brasil.

Gastrotheca albolineata: Izecksohn \& Carvalho-e-Silva, 2001: 37. Sachsse, Izecksohn \& Carvalho-e-Silva, 1999: 401.

Os exames do holótipo e de um topotipo da Serra dos Órgãos confirmaram a identidade dos demais exemplares atribuídos a esta espécie. A presença de um par de destacadas faixas laterais brancas (amarelas anteriormente) percorrendo do focinho aos flancos separa esta espécie das demais da mesma procedência. Faixas brancas percorrem também o bordo externo do tarso e acima da abertura cloacal. Finas estrias brancas delimitam figuras simétricas no dorso e nas patas. A pele no dorso da cabeça é aderente mas não há co-ossificação. Em vida, a cor geral é ver-

Revista Brasileira de Zoologia 25 (1): 100-110, March, 2008 


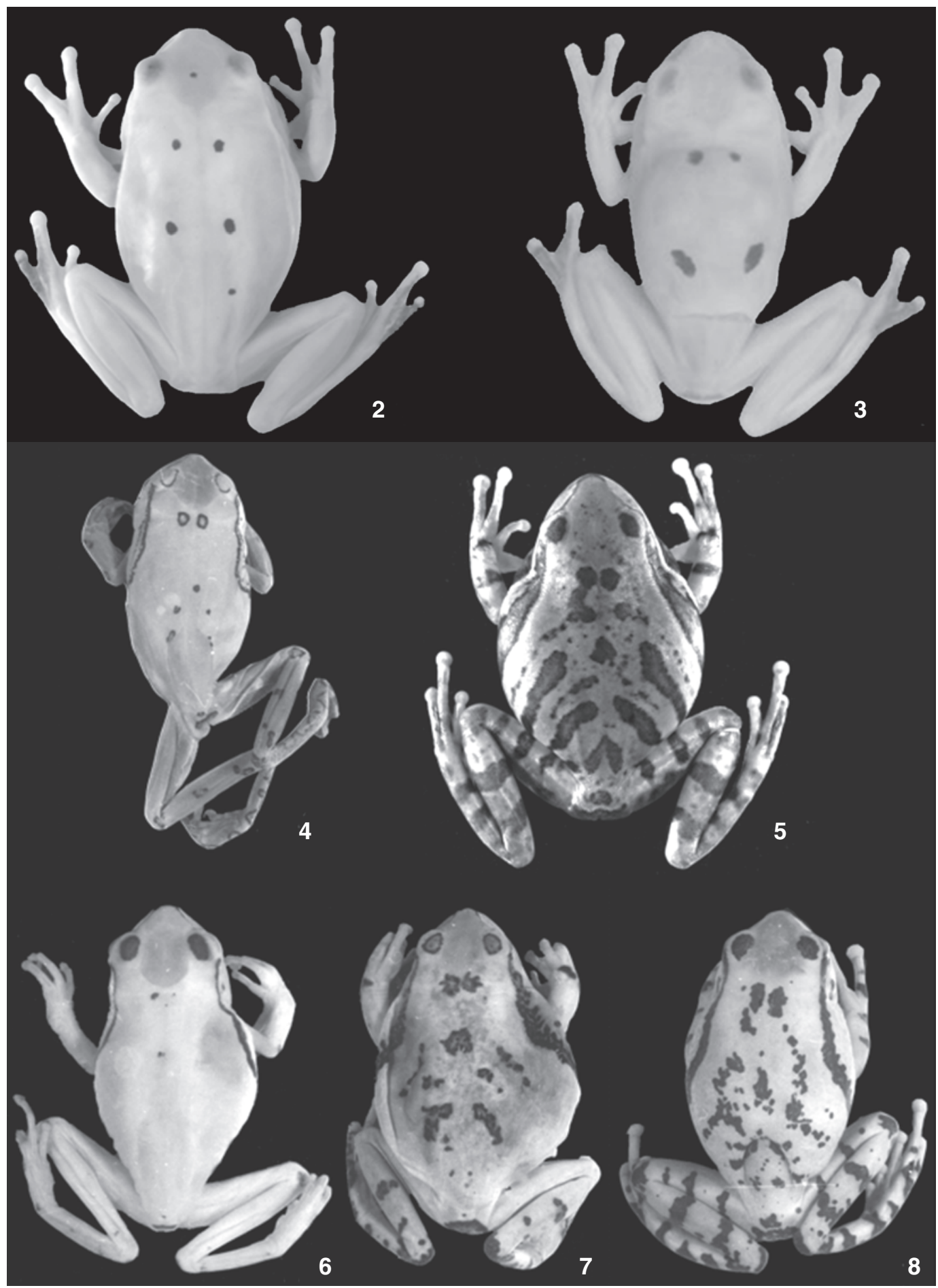

Figuras 2-8. (2-3) Gastrotheca albolineata, dorsos: (2) macho adulto, ZUFRJ 6456, CRC = 60 mm, Teresópolis, Estado do Rio de Janeiro; (3) fêmea adulta, ZUFRJ 7050, CRC = 60 mm, Teresópolis, Estado do Rio de Janeiro; (4-8) G. ernestoi, dorsos: (4) fêmea adulta, AL 969, $\mathrm{CRC}=60 \mathrm{~mm}$, Bonito, Serra da Bocaina, Estado de São Paulo (Holótipo de G. viridis); (5) macho adulto, UNIRIO 2534, CRC = 57 mm, Teresópolis, Estado do Rio de Janeiro; (6) jovem, El s/no, CRC = 29 mm, idem, idem.; (7) fêmea adulta, MNRJ 19328, CRC = 72 mm, Itatiaia, Estado do Rio de Janeiro; (8) fêmea adulta, MNRJ 19330, CRC = 70 mm, Teresópolis, Estado do Rio de Janeiro.

Revista Brasileira de Zoologia 25 (1): 100-110, March, 2008 


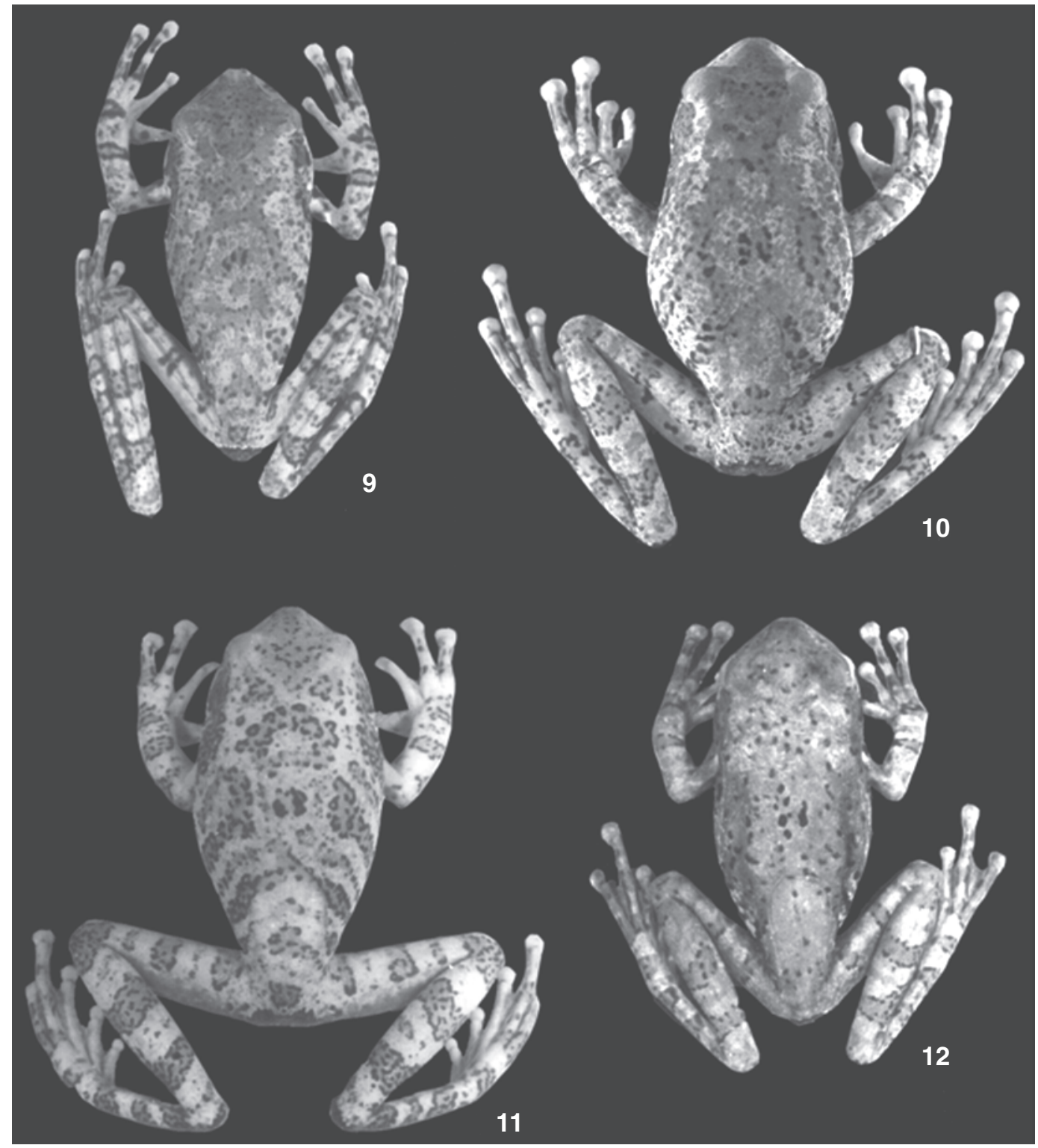

Figuras 9-12. Gastrotheca fulvorufa, dorsos: (9) macho adulto, MNRJ 19323, CRC = 63 mm, Teresópolis, Estado do Rio de Janeiro; (10) macho adulto, ZUFRJ 8287, CRC = $68 \mathrm{~mm}$, idem, idem; (11) fêmea adulta, El s/nº, CRC = $72 \mathrm{~mm}$, idem, idem; (12) fêmea adulta, El s/ $\mathrm{n}^{\circ}, \mathrm{CRC}=72 \mathrm{~mm}$, idem, idem.

de-folha, com dois pares de pintas negras ou pardo-escuras, um pós-nucal e outro sobre o sacro, formando um trapézio. A largura do disco do terceiro dedo é maior que o diâmetro do tímpano.

\section{Gastrotheca ernestoi A. Miranda-Ribeiro, 1920}

Figs $4-8,14,17,20,23,28,29,33$

Gastrotheca ernestoi A. Miranda-Ribeiro, 1920: 323. Localidadetipo: Macaé, Estado do Rio de Janeiro, Brasil; Izecksohn \& Carvalho-e-Silva, 2001: 37.
Gastrotheca viridis Lutz \& Lutz, 1939: 81. Localidade-tipo: Bonito, Serra da Bocaina, Estado de São Paulo, Brasil.

Gastrotheca microdisca (parte); Cochran, 1955: 50; Duellman, 1984: 304.

O exame do holótipo confirmou a identidade dos exemplares provenientes da Serra dos Órgãos e reunidos sob este nome. Trata-se de espécie grande, sem co-ossificiação da pele do crânio, mas com aderência desta nos exemplares recém fixados. Dorsalmente e lateralmente apresenta manchas ou fai-

Revista Brasileira de Zoologia 25 (1): 100-110, March, 2008 
xas oblíquas, simétricas, pardo-escuras ou negras, que contrastam com o fundo bege ou verde. Sobre as pálpebras superiores há um par de manchas redondas ou ocelos negros ou pardoescuros, havendo geralmente um par de manchas semelhantes pós-nucais. As faixas escuras laterais podem apresentar margens claras. A largura do disco do terceiro dedo é maior que o diâmetro do tímpano.

\section{Gastrotheca fulvorufa (Andersson, 1911)} Figs $9-12,15,18,21,24,30,31,34$

Nototrema fulvorufa Andersson, 1911: 3. Localidade-tipo: Santos, Estado de São Paulo, Brasil.

Hyla parkeri DeWitte, 1930a: 226 (não Hyla parkeri Gaige, 1929). Localidade-tipo: Alto da Serra, São Paulo.

Hyla parkeriana DeWitte, 1930b:102 (novo nome para Hyla parkeri DeWitte).

Gastrotheca fulvo-rufa: A. Miranda-Ribeiro, 1926: 110.

Gastrotheca microdisca [sic] (parte); Cochran, 1955: 50; Duellman, 1984: 304.

Gastrotheca fulvorufa; Izecksohn \& Carvalho-e-Silva, 2001: 37.

Não examinamos o holótipo, mas a descrição e figuras originais não nos deixaram dúvidas quanto à identidade dos exemplares reunidos da Serra dos Órgãos. Trata-se de espécie grande sem capacete ou co-ossificação na cabeça, mas com aderência da pele na zona mediana do crânio nos indivíduos vivos ou recentemente coletados. Os exemplares examinados apresentam um pequeno e destacado grupo de grânulos negros no centro do dorso a meia distância entre a extremidade do focinho e a abertura cloacal. As figuras pardas que compõem a ornamentação dorsal estão sempre delimitadas por filas de pequenos pontos negros. A cor fundamental dorsal, em vida, varia do verdemaçã (juvenis) ao bege avermelhado (adultos). A largura do disco do terceiro dedo é maior que o diâmetro do tímpano.

\section{Estudo comparativo de exemplares das três espécies de Gastrotheca obtidos na Serra dos Órgãos}

Os maiores exemplares entre o material examinado apresentaram comprimento rostro-cloacal para G. albolineata $=60$ $\mathrm{mm}$ (macho e fêmea), para G. ernestoi $=75 \mathrm{~mm}$ (fêmea), e para G. fulvorufa $=80 \mathrm{~mm}$ (fêmea).

Gastrotheca albolineata (Figs 2 e 3) e G. ernestoi (Figs 4-8) possuem corpo relativamente mais largo que o de G. fulvorufa (Figs 9-12).

Os focinhos de G. albolineata (Fig. 13) e de G. ernestoi (Fig. 14) têm perfis quase truncados; o de G. fulvorufa (Fig. 15) mostra perfil arredondado.

As pálpebras superiores de G. ernestoi exibem um ocelo ou elipse, negro ou pardo-escuro, compacto ou com o centro claro (Figs 4-8). As pálpebras de G. albolineata (Figs 2 e 3) e de G. fulvorufa (Figs 9-12) não mostram esse tipo de ornamentação.

Gastrotheca albolineata mostra-se dorsalmente verde intenso em vida, apresentando dois pares de pintas pardas ou
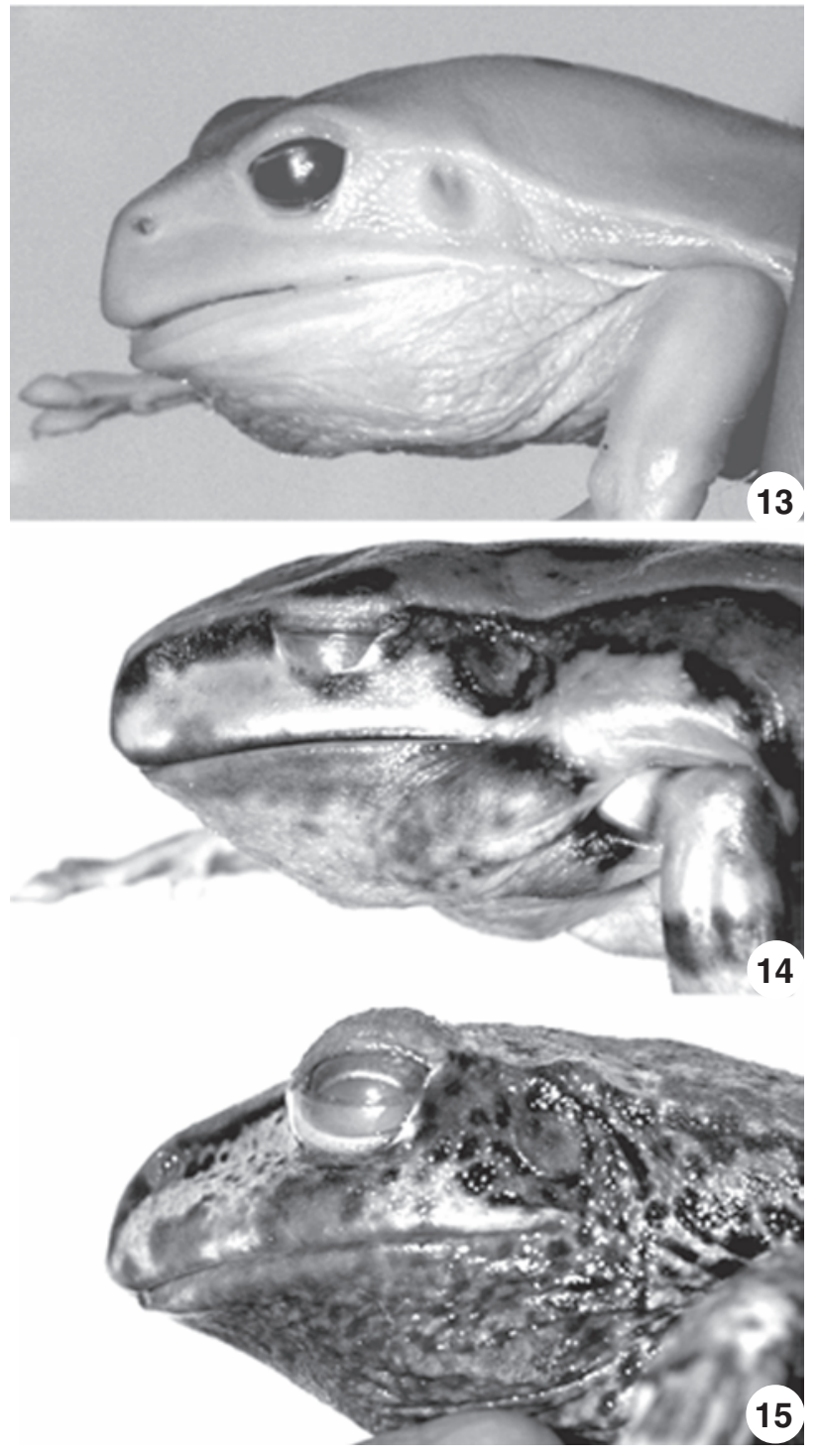

Figuras 13-15. Perfil dos focinhos de exemplares de Gastrotheca: (13) G. albolineata, ZUFRJ 6456; (14) G. ernestoi, UNIRIO 2534; (15) G. fulvorufa ZUFRJ 8267.

negras, dispostas como os cantos de um trapézio, o primeiro mais aproximado e situado sobre as supra-escápulas, e o segundo, com as pintas mais afastadas entre si, situado pouco adiante do sacro (Figs 2, 3) e, por vezes, uma pinta sobre a cabeça; nesta espécie podem ser notadas, em vida, finas estrias brancas que se dispõem como se marginassem indistintas figuras simétricas no dorso e bandas nas pernas (Fig. 27). G. ernestoi (Figs 48) mostra na cabeça, dorso e patas coloração fundamental bege ou pardo claro, por vezes e ao menos em parte verde claro; atrás da cabeça há um par de manchas negras, às vezes fundidas, compactas ou com o centro mais claro; o dorso do tronco

Revista Brasileira de Zoologia 25 (1): 100-110, March, 2008 


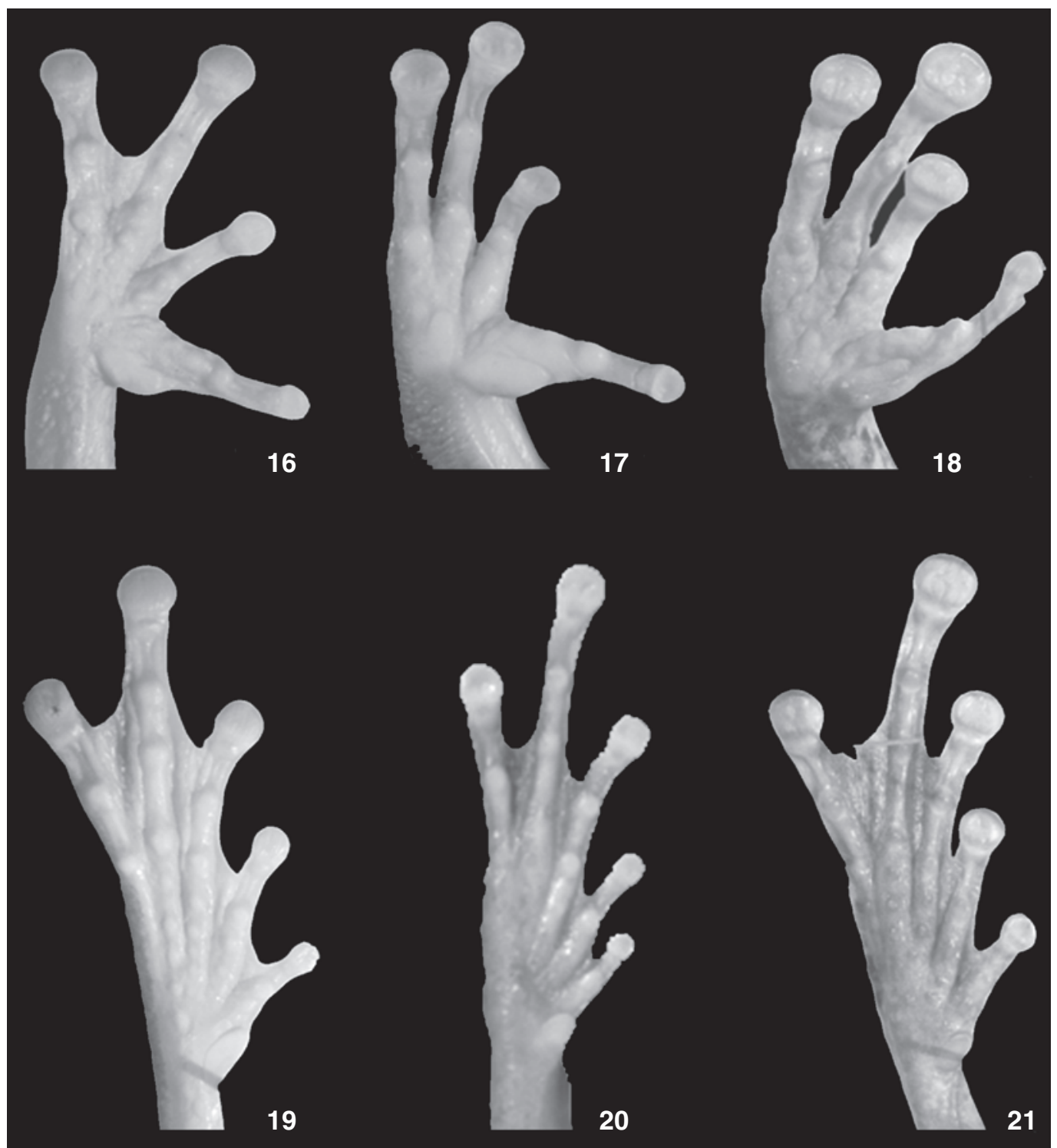

Figuras 16-21. Mãos (face palmar) e pés (face plantar) de exemplares de Gastrotheca: (16 e 19) G. albolineata, ZUFRJ 6456; (17 e 20) G. ernestoi, UNIRIO 2534; (18 e 21) G. fulvorufa, ZUFRJ 8287.

exibe faixas negras ou pardo-escuras, oblíquas, que divergem para trás. G. fulvorufa (Figs 9-12) tem sua coloração dorsal fundamental pardo-avermelhada, (verde-clara em juvenis), com figuras simétricas pardas, irregulares em suas formas, divergentes para trás, marginadas por pontos ou grânulos negros; diversos outros grânulos negros estão presentes entre estas figuras e há um pequeno conjunto de tubérculos, também negros, situados no centro do dorso a meia distância entre a extremidade do focinho e a abertura cloacal.

Gastrotheca albolineata (Figs 26-27) exibe nos loros e flancos apenas uma faixa branca (em vida amarelada no focinho), marginada por estria castanha, que percorre o lábio superior, passa sob o olho, sob tímpano, sobre a inserção do úmero e vem terminar no flanco pouco à frente da região inguinal; G. ernestoi (Figs 28-29) apresenta uma estreita faixa esbranquiçada desde a extremidade do focinho até o úmero, passando pelo lábio superior, sob o olho e sob o tímpano, e também uma estria pardoclara, marginada ventralmente por uma faixa marrom ou negra, desde o canto rostral, passando pela borda da pálpebra, até o flanco onde se encurva para o ventre; G. fulvorufa (Figs 30-31) não possui, nos loros e flancos, estrias ou faixas claras longitudinais, mas mostra uma mancha marrom sob o olho e outra desde

Revista Brasileira de Zoologia 25 (1): 100-110, March, 2008 

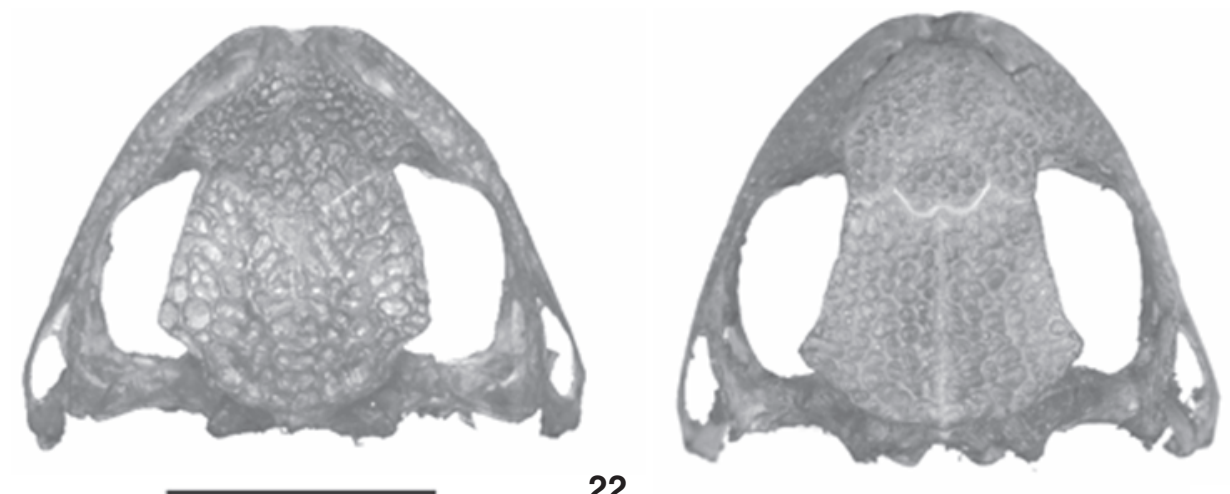

22
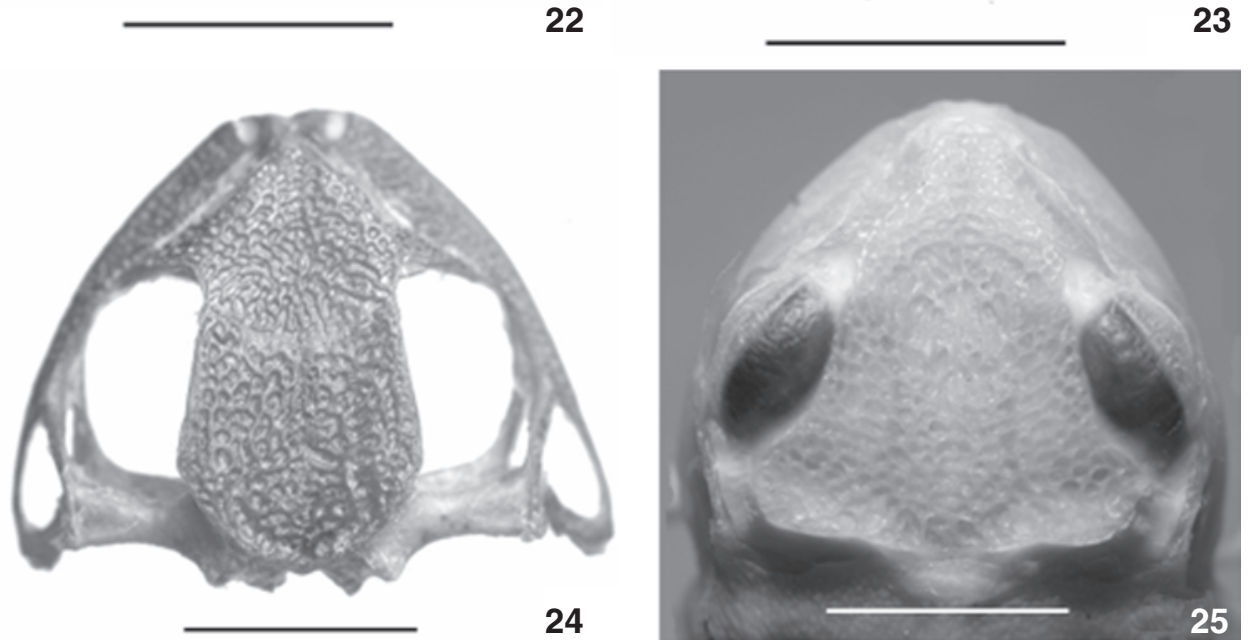

Figuras 22-25. Vistas dorsais de crânios de exemplares de Gastrotheca mostrando as diferentes formas dos frontoparietais: (22) G. albolineata, ZUFRJ 3416; (23) G. ernestoi, UNIRIO 2534; (24) G. fulvorufa, El s/nº; (25) G. sp., El s/nº (Ilha de São Sebastião, Estado de São Paulo).

o bordo posterior do olho até a comissura labial, encobrindo o tímpano, e nos flancos manchas e faixas marrons ou negras, irregulares.

Em vida a íris de G. albolineata se apresenta amarela, em G. ernestoi a íris é parda e em G. fulvorufa ela é cor de ferrugem.

Em G. albolineata e G. ernestoi a pele no dorso se mostra finamente granulosa, enquanto em $G$. fulvorufa a pele apresenta diversos grânulos maiores.

As membranas das mãos e pés de G. albolineata são mais extensas do que as das duas outras espécies (Figs 16-21).

Nas espécies estudadas observou-se aderência da pele a trechos da superfície do crânio, mas isto desapareceu após algum tempo de manutenção dos exemplares nos líquidos conservadores, deixando então a pele solta. Ela é decorrente da superfície rugosa dos ossos do crânio que têm contato com a pele, como os frontoparietais, esfenoetmóides, nasais e as partes superficiais dos maxilares e escamosais. Essa rugosidade ou ornamentação se mostra como um conjunto de rugas que formam retículos e deixam cavidades rasas de contorno redondo entre elas. Os frontoparietais são os ossos do crânio com maior área de contacto com a pele e sua forma difere entre as espécies encontradas na Serra dos Órgãos. Eles possuem flanges laterais angulosas que se projetam para as órbitas em maior ou menos extensão. Em G. albolineata as flanges dos frontoparietais são algo mais expandidas do que nas outras duas espécies, apresentando lateralmente ângulos mais acentuados e retículos maiores (Fig. 22). Os frontoparietais de G. ernestoi são algo menos expandidos, com ângulos laterais menores (Fig. 23) e os frontoparietais de G. fulvorufa têm seu contorno convexo, quase sem ângulos (Fig. 24). Nenhuma das três espécies encontradas na Serra dos Órgãos mostra os frontoparietais formando o bordo posterior das órbitas como em Gastrotheca sp. (Fig. 25), do litoral do Estado de São Paulo.

A tíbia, por ser retilínea e ter suas extremidades fora do tronco, é o segmento da perna que permite medição mais precisa e é proporcional, em comprimento, aos demais segmentos dos membros posteriores, dando idéia da extensão relativa da pata. A relação CT/CRC observada em quatro exemplares adultos de cada espécie mostrou para G. albolineata variação entre $45 \%$ e $48 \%$, para G. ernestoi variação de $48 \%$ a $49 \%$ e para $G$. fulvorufa variação de $54 \%$ a $62 \%$.

Nas três espécies examinadas, os discos do terceiro dedo 


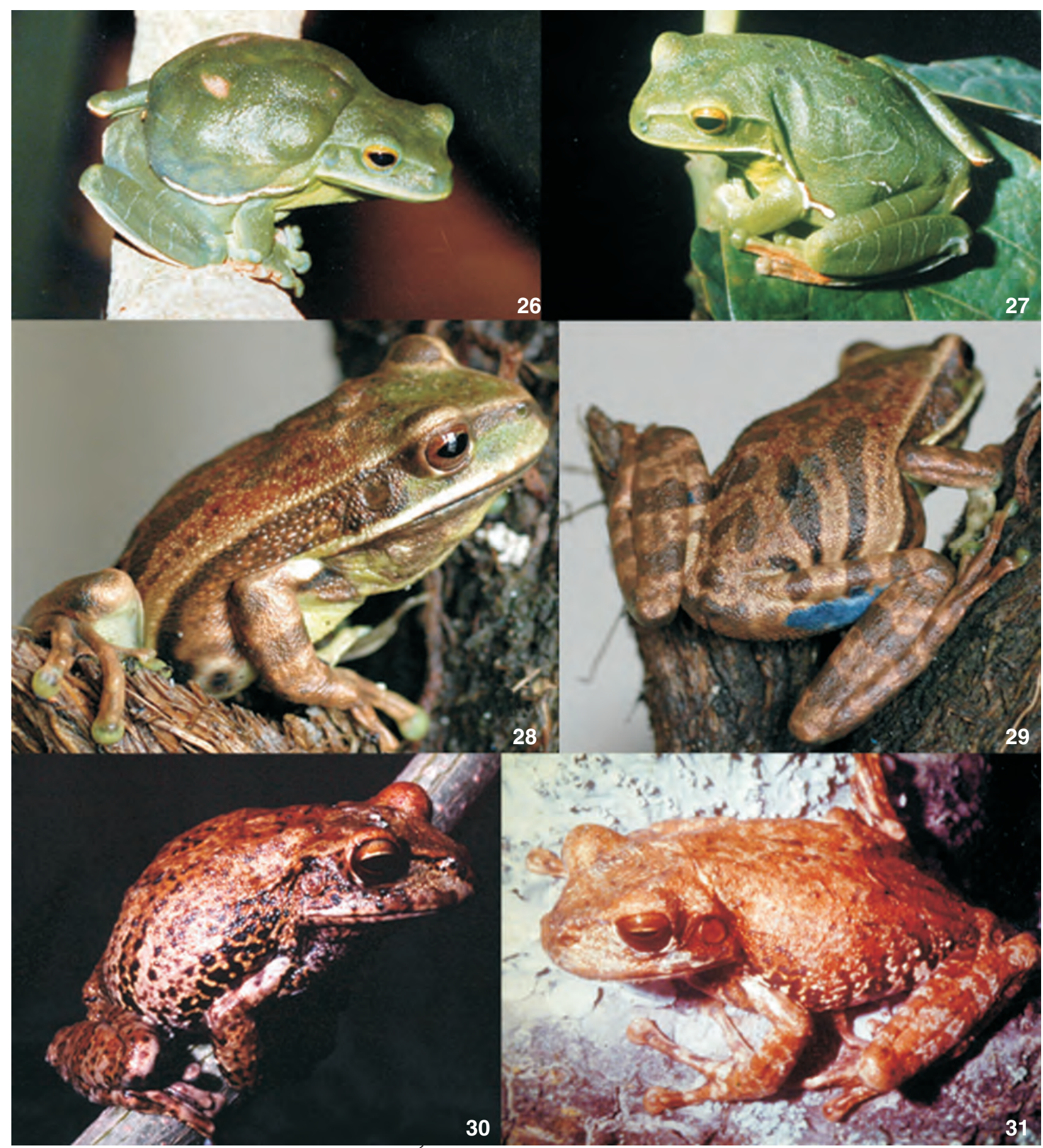

Figuras 26-31. Exemplares de Gastrotheca da Serra dos Órgãos, vivos: (26) fêmea de G. albolineata, ZUFRJ 7050; (27) macho de G. albolineata, ZUFRJ 6456; (28 e 29) macho de G. ernestoi, UNIRIO 2534; (30) macho de G. fulvorufa, ZUFRJ 8287; (31) fêmea de G. fulvorufa, El s/nº.

e do quarto artelho mostraram suas larguras iguais ou pouco maiores que o diâmetro transversal dos tímpanos.

A vocalização de $G$. albolineata (oito registros), com duração entre 2,0 e 4,0 segundos, consta de cinco a 14 notas graves, com duração média de $0,07 \mathrm{~s}$, que se repetem a cada $0,30 \mathrm{~s}$, podendo ter notas introdutórias que são emitidas em escala crescente de intensidade, lembrando o timbre de uma galinha quando estressada. Cada nota é formada por pulsos com freqüência

Revista Brasileira de Zoologia 25 (1): 100-110, March, 2008 


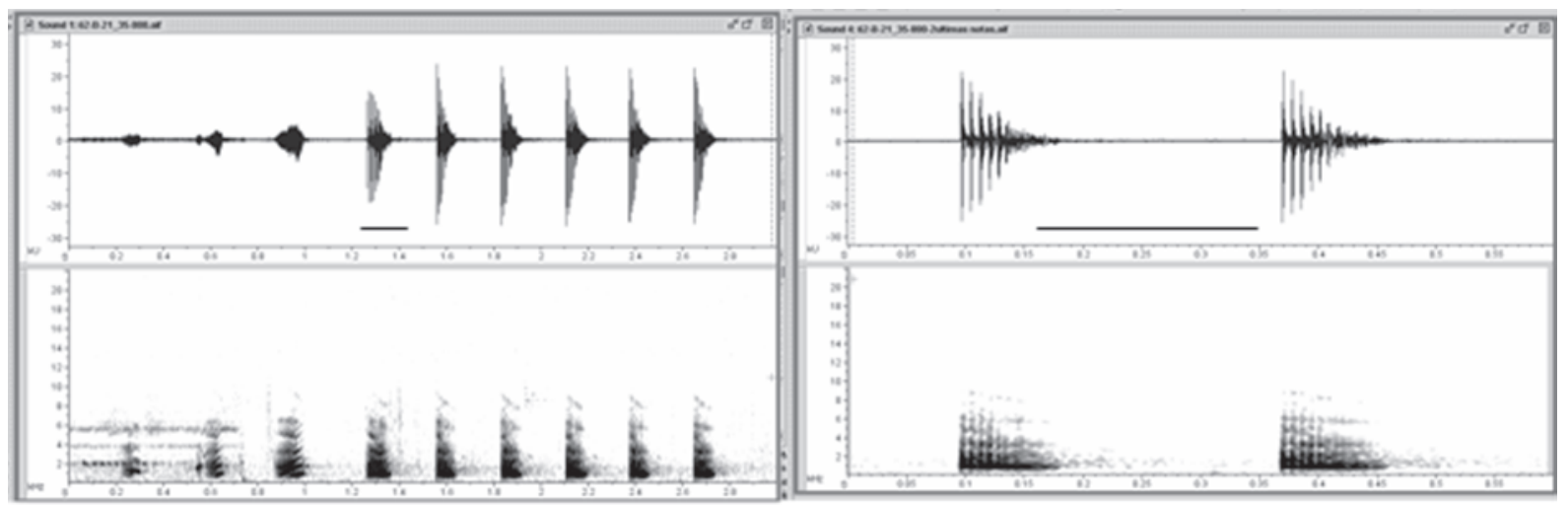

32

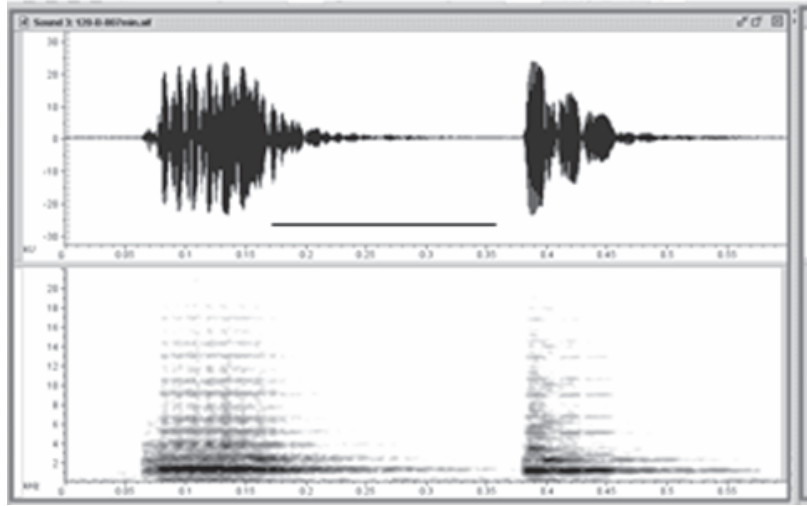

34

Figuras 32-35. Oscilogramas e sonogramas de espécies de Gastrotheca da Serra dos Órgãos: (32 e 33) G. albolineata; (34) G. ernestoi; (35) G. fulvorufa. (Escalas =0,2 s).

dominante entre $1200 \mathrm{hz}$ e $1700 \mathrm{hz}$ e freqüência fundamental entre 650 hz e 800 hz. A vocalização de G. ernestoi (dois registros) com duração de $0,4 \mathrm{~s}$, é algo estridente, sugerindo o grito do pássaro cotingídeo "araponga" ou "ferreiro" (Procnias nudicolis) quando ouvido ao longe e consta geralmente de duas notas de igual intensidade, a primeira $(0,18 \mathrm{~s})$ mais longa que a segunda $(0,11 \mathrm{~s})$, emitidas com intervalo de $0,30 \mathrm{~s}$. A primeira nota é formada por cerca de sete pulsos semelhantes e a segunda por três pulsos cada um menos intenso que o anterior. As duas notas apresentam estrutura de harmônicos, sendo o dominante ( = fundamental), com freqüência em torno de 1119 hz. Embora o canto mais freqüente apresente duas notas, um dos autores (SPCS) ouviu vocalizações no ambiente com até oito notas. G. fulvorufa (quatro registros) vocaliza emitindo notas sonoras, musicais, algo flauteadas, com seu timbre sugerindo, à distância, a voz de Aplastodiscus albofrenatus (A. Lutz), e a vocalização com duração media $0,5 \mathrm{~s}$, consta de duas notas não formadas por pulsos, com a mesma intensidade, com $0,16 \mathrm{~s}$ a primeira e $0,06 \mathrm{~s}$ a segunda, emitidas com intervalo de 0,4 s. Apresentam estrutura de harmônicos com o harmônico dominante em torno de 1785 hz e o fundamental em $904 \mathrm{hz}$.

Revista Brasileira de Zoologia 25 (1): 100-110, March, 2008
Os exemplares de G. fulvorufa encontrados em repouso estavam sempre refugiados em bromélias, tanto os jovens como os adultos. Dois indivíduos de G. ernestoi mantidos em terrários, um da área estudada e o outro de Itatiaia, igualmente no Estado do Rio de Janeiro, demonstraram também sua preferência por bromélias como local de abrigo. Nenhum indivíduo de $G$. albolineata, tanto adulto como filhote, demonstrou qualquer afinidade com as bromeliáceas, mesmo em terrários, preferindo repousar sempre sobre folhas verdes.

\section{Exemplares examinados}

Gastrotheca albolineata (Lutz \& Lutz) - Estado do Rio de Janeiro, Teresópolis: AL 1778 (holótipo de Hyla albolineata), jovem; MNRJ 3223, jovem adulto; ZUFRJ 3437, jovem; ZUFRJ 6456, macho adulto; ZUFRJ 6457, jovem adulto; ZUFRJ 6923, macho adulto (crânio retirado); ZUFRJ 7050, fêmea adulta; ZUFRJ 7051-66, recém-nascidos em terrário; ZUFRJ 9416, macho adulto (diafanizado e crânio retirado). Cachoeira de Macacú: RURAL 020, macho adulto.

Gastrotheca ernestoi Miranda-Ribeiro - Estado do Rio de Janeiro, Macaé: MZUSP 238 (holótipo de Gastrotheca ernestoi), fêmea adulta. Teresópolis: MNRJ 19325, macho adulto; MNRJ 
19326, jovem; MNRJ 19327, adulto jovem; MNRJ 19329, fêmea adulta; MNRJ 19330, fêmea adulta; MNRJ 19331, jovem; EI s/no, jovem; UNIRIO 2534, macho adulto (crânio retirado). Itatiaia: MNRJ 19328, fêmea adulta; EI s/nº macho adulto (crânio retirado). Estado de São Paulo, Bonito (Serra da Bocaina): AL 969 (holótipo de Gastrotheca viridis), fêmea adulta.

Gastrotheca fulvorufa (Andersson) - Estado do Rio de Janeiro, Teresópolis: AL s/nº, macho adulto; MNRJ 19323, macho adul-

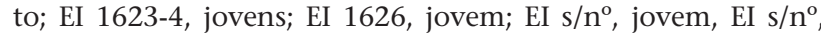
jovem; EI s/no fêmea adulta; EI s $/ n^{\circ}$, fêmea adulta, ZUFRJ 621, macho adulto; ZUFRJ 4184-5, jovens aparentemente recém-natos; ZUFRJ 8287, macho adulto. Nova Friburgo: AL 3562, fêmea.

Gastrotheca sp. - Estado de São Paulo, Ilha de São Sebastião: EI $s / n^{\circ}$, fêmea adulta.

\section{DISCUSSÃO}

Andersson (1910) quando descreveu e figurou Nototrema microdiscus, informou que a espécie apresentava a derme, na cabeça, completamente envolvida em uma ossificação craniana debilmente rugosa estendendo-se até uma linha semicircular, correndo entre os bordos posteriores das órbitas e com a convexidade posterior, sendo que somente as pálpebras superiores, a ponta do focinho e uma estreita faixa da margem livre do lábio superior eram moles. ANDERsson (loc. cit.) assinalou, também, que os discos dos dedos eram pequenos, não maiores do que a metade do tímpano e os dos artelhos eram ainda menores. Posteriormente, o mesmo autor (ANDERsson 1911), ao descrever Notorema fulvorufa, relatou que a derme da cabeça era livre de ossificação craniana e que os discos do terceiro e quarto dedos, assim como os dos artelhos, eram tão grandes quanto o tímpano.

Em todos os exemplares de Gastrotheca examinados procedentes da Serra dos Órgãos, os discos do segundo e terceiro dedos, bem como do terceiro e quarto artelhos, eram iguais ou maiores que o diâmetro do tímpano e a pele sobre o crânio não se mostrou co-ossificada. Entretanto, tivemos a oportunidade de examinar uma fêmea de uma espécie de Gatrotheca com 67 $\mathrm{mm}$ de comprimento rostro-cloacal, com a bolsa formada e ovócitos desenvolvidos nos oviductos, procedente da Ilha de São Sebastião, no litoral do Estado de São Paulo, mostrando intensa aderência entre a derme e o crânio até atrás das órbitas, encobrindo os proóticos (Fig. 25). Este exemplar estava com seu colorido desbotado e mostrava discos grandes nos dedos e artelhos, o que não nos permitiu sua identificação como $G$. microdiscus. Entre as espécies brasileiras conhecidas de Gastrotheca, apenas fissipes e microdiscus são referidas como possuindo envolvimento da derme com o crânio e, portanto, consideramos inaplicável o nome microdiscus a qualquer das três espécies aqui registradas para a região da Serra dos Órgãos.

Um exemplar juvenil de G. ernestoi (Fig. 6) obtido no Parque Nacional da Serra dos Órgãos era verde e possuía como padrão de colorido apenas dois ocelos sobre as pálpebras, uma pequena pinta negra no dorso atrás da cabeça, e estria negra curvada percorrendo os flancos, reproduzindo o colorido de $G$. viridis (Fig. 4) dado por Lutz \& LuTZ (1939).

A presença de ovos grandes e pouco numerosos na bolsa dorsal já foi constatada em espécies brasileiras do gênero para G. fissipes (Boulenger 1888, Miranda-Ribeiro 1926), G. microdiscus (Andersson 1910), G. ernestoi (Miranda-Ribeiro 1920, 1926) e $G$. albolineata (SACHSSE et al. 1999). Ainda não foi registrado, porém, exemplar de G. fulvorufa com ovos na bolsa dorsal, mas foram obtidos dois exemplares muito jovens da espécie completamente metamorfoseados, com comprimento rostro-cloacal de $20 \mathrm{~mm}$, encontrados próximos um do outro, sugerindo que o abandono da bolsa materna era recente.

A dificuldade em se obter exemplares e a relativa riqueza de espécies de Gastrotheca na área estudada, o que deve ocorrer também em outros segmentos da floresta atlântica, permite prever que outras espécies deste gênero devem existir nas matas das serras litorâneas brasileiras, aguardando suas descobertas.

\section{CONCLUSÕES}

A Serra dos Órgãos, no Estado do Rio de Janeiro, abriga ao menos três distintas espécies do gênero Gastrotheca Fitzinger: G. albolineata (Lutz \& Lutz), G. ernestoi Miranda-Ribeiro e G. fulvorufa (Andersson), sendo que o nome Gastrotheca microdiscus (Andersson) não pode ser aplicado a nenhuma delas por se tratar de espécie diversa, ainda não encontrada na região, descrita como possuidora de derme envolvida em uma ossificação craniana debilmente rugosa e de discos digitais com sua largura correspondendo à metade da do tímpano.

Gastrotheca albolineata é reconhecível por apresentar, em vida, cor intensamente verde, ornamentação dorsal escura constituída apenas por quatro pintas negras ou pardas dispostas como os cantos de um trapézio, as posteriores mais afastadas entre si, e finas estrias brancas dispostas como se marginassem figuras indistintas no dorso e patas, e como ornamentação lateral uma faixa branca (amarela em seu início), marginada de marrom, que percorre o lábio superior, passando sob o olho e vindo terminar no meio do flanco. Essa espécie igualmente se destaca por mostrar uma faixa marrom ferrugem sob o ânus e em cada tarso, que é marginada por estria branca e se estende, no lado externo dos pés, desde a articulação tíbio-tarsal até o quinto e o quarto artelhos.

Gastrotheca ernestoi se destaca, entre as espécies estudadas, por apresentar um par de ocelos ou elipses escuras sobre as pálpebras e faixa labial clara, além de uma faixa lateral escura que percorre o focinho e os flancos, onde se curva para o ventre. A ornamentação sobre o dorso consta de faixas ou manchas negras ou pardo-escuras e não são visíveis grânulos negros dispersos ou formando séries.

Gastrotheca fulvorufa não mostra ocelos palpebrais, faixa branca labial nem faixa clara lateral, mas ostenta manchas escuras suboculares e postoculares. A face dorsal da cabeça e tronco exibe manchas ou faixas pardacentas irregulares, simétricas, sempre delimitadas por séries de pontos ou grânulos negros, que também se distribuem entre as manchas. No centro da face dorsal há um pequeno grupo destacado de tubérculos negros.

Revista Brasileira de Zoologia 25 (1): 100-110, March, 2008 


\section{AGRADECIMENTOS}

Por suas colaborações em alguma etapa da preparação deste trabalho, agradecemos a Ana Carolina O. de Q. Carnaval, Ana M.P.T. de Carvalho e Silva, Antenor L. de Carvalho (in memoriam), Carlos A.G. da Cruz, Cyro de L. Dias Netto, Márcia dos R. Gomes, Paulo E. Vanzolini, Oswaldo L. Peixoto, Thiago S. Soares, Ulisses Caramaschi e Werner C.A. Bokermann (in memoriam). Somos especialmente gratos a Direção do Parque Nacional da Serra dos Órgãos pelas facilidades proporcionadas para a realização dos trabalhos de campo.

\section{LITERATURA CITADA}

Andersson, L.G. 1910. A new lizard and a new frog from Parana. Arkiv för Zoologi 6 (9): 1-11.

ANDERSSON, L.G. 1911. A new Leptodactylus and a new Nototrema from Brazil. Arkiv för Zoologi 7 (17): 1-6.

Boulenger, G.A. 1888. On some reptiles and batrachians from Iguarasse, Pernambuco. Annals and Magazine of Natural History, Séries 6, 2 (7): 40-43.

Cochran, D.M. 1955. Frogs of southeastern Brazil. Bulletin of United States National Museum 206: 1-423.

DeWitte, G.F. 1930a. Liste des reptiles et batraciens recoltes au Bresil par la Mission Massart (1922-23) et description de sept nouvelles espécies. In: Une mission biologique Belge au Bresil (Aout 1922-Mai 1923), vol. 2, p. 214-230.

DeWitte, G.F. 1930b. Hyla parkeriana n. nom. Revue de Zoologie et de Botanique Africaines 20: 202.

Received in 26.VI.2007; accepted in 14.II.2008.
Duellman, W.E. 1984. Taxonomy of Brazilian Hylid Frogs of the Genus Gastrotheca. Journal of Herpetology 18 (3): 302-312.

Frost, D.R. 2007. Amphibian species of the world: an online reference. Version 5.0. New York, American Museum of Natural History, Electronic Database available at http://research. amnh.org/amphibia/index.php [Accessed in 01.II.2007]

Izecksohn, E. \& S.P. Carvalho-e-Silva. 2001. Anfíbios brasileiros que carregam ovos nas costas. Aquarium 30: 36-37.

Izecksohn, E.; S.P. Carvalho-e-Silva \& I. Deiss. 2005. O osteocrânio de Proceratophrys boiei (Wied-Neuwied), P. appendiculata (Günther), P. melanopogon (Miranda-Ribeiro) e P. laticeps Izecksohn \& Peixoto (Anura, Leptodactylidae). Revista Brasileira de Zoologia 22 (1): 225-229.

Lutz, A. \& B. Lutz 1939. New hylidae from Brazil. Anais da Academia Brasileira de Ciências 11: 67-89.

Miranda-Ribeiro, A. De. 1920. As hylas coelonotas do Museu Paulista. Revista do Museu Paulista 12: 321-328.

Miranda-Ribeiro, A. DE. 1926. Notas para servirem ao estudo dos gymnobatrachios (Anura) Brasileiros. Archivos do Museu Nacional do Rio de Janeiro 27: 1-227.

Sachsse, R; E. Izecksohn \& S.P. carvalho-e-Silva. 1999. The Systematic Status of Hyla albolineata Lutz and Lutz, 1939 (Amphibia: Anura: Hylidae). Herpetologica 55 (3): 401-406.

Weber, L.N.; L.A.P. Gonzaga \& S.P. Carvalho-e-Silva. 2005. A new species of Physalaemus Fitzinger, 1826 from the lowland Atlantic Forest of Rio de Janeiro State, Brazil (Amphibia, Anura, Leptodactylidae). Arquivos do Museu Nacional do Rio de Janeiro 63 (4): 677-684. 